\title{
Pedestrian Accidents - Actual Trend in the Czech Republic
}

\author{
I. Kučerová*, B. Sulíková, K. Paráková, K. Poláčková \\ Transport Research Centre, Brno, Czech Republic \\ *Corresponding author: irena.kucerova@cdv.cz
}

DOI: $10.2478 / v 10158-012-0041-0$

\begin{abstract}
There are many factors that play a role in pedestrian and motor vehicle collisions. This paper explores some of the factors on the basis of police records, involving pedestrian accidents in the Czech Republic in the period between 2008 and 2012; the sample consisting of 19454 participants. This paper explores the impact of pedestrian factors (age, gender, behaviour, injury severity, etc.) and other road/environmental factors (visibility, accident location, weather, and so on) on pedestrian collisions. The purpose of the crosstabs analysis was to determine if there is a relationship in these selected factors; the relevance of the factors was examined in a group, not independently. The analysis identified the children and elderly as the most vulnerable road users, with the emphasis given to higher mortality of elderly people and higher tendency of children to violate traffic rules. The majority of pedestrian collisions took place in urban areas, at pedestrian crossings, with the exception of collisions involving children that took place when crossing between parked vehicles (including bus stops) and on unprotected road sections without pedestrian crossings. The results also show that the majority of fatal collisions occurred at night, and that alcohol-impaired walking is a major problem especially for the population of middleaged male pedestrians.
\end{abstract}

KEY WORDS: Pedestrian collision, pedestrian safety, accident location.

\section{INTRODUCTION}

This article is an explorative study focused on pedestrian accidents in the Czech Republic. We focus on factors that have been identified as significant in studies with a similar topic. Then we used them for purposes of our study. As far as pedestrians are the most vulnerable road users (VRUs), the analysis of the pedestrian collisions is an important traffic safety concern. In the Czech Republic the total number of pedestrian collisions is constantly decreasing over time, but on the other hand, pedestrian accidents are still on the increase in urban areas at night-time, and the most vulnerable groups still include the children and the elderly (Police Statistics of the Czech Republic, 2012).

Pedestrians pose almost no threat to other road users, yet suffer almost one quarter of total casualties killed or seriously injured on the roads (PACTS, 2013). The study of overall trends in EU shows that, although pedestrian fatalities in Europe present a decreasing trend, pedestrian fatality rates are still on the increase in Southern European countries, as well as in the new EU Member States, which includes the Czech Republic as well (Yannis et al., 2011). In comparison to other countries of EU, e.g. Germany, Ireland and the United Kingdom, the decrease has been higher than 47\% from 2001 to 2010 (49\% for the Czech Republic). The rate in Czech Republic was 16 fatalities per million inhabitants 
in 2010 (Pace et al., 2012). The situation is more or less the same throughout the world (WHO, 2013).

We focused on the factors that were subjects of interest in similar studies. The most common factors that are used to characterize pedestrian accidents and became central to our research are age and gender of the pedestrians (Holland \& Hill, 2007; Fontaine \& Gourlet, 1997), with the strong emphasis given to the higher vulnerability of children and the elderly (Yannis et al., 2011, Zhuang \& Wu 2011, Fontaine \& Gourlet, 1997).

According to age, children and elderly are the most vulnerable road users. The highest fatality rate occurs for pedestrians older than 75 years of age. Although a high percentage of children fatalities were pedestrians, they only represent $4 \%$ of the total pedestrian fatalities in 2010 (WHO, 2013). The proportion of pedestrian fatalities for children varies widely among the EU countries (Pace et al., 2012), so we try to identify the situation of this age group in the Czech Republic and expect that fact of vulnerable groups will be confirm also in our study.

Another observed factor refers to the higher involvement of males in pedestrian collisions (e.g. Beck et al., 2007; Al-Shammari et al., 2009). In 2010 there were more male than female pedestrian fatalities in almost every EU country. Total distribution shows that 64\% of pedestrian fatalities were male and 36\% were female (Pace et al., 2012). The results will be compared to our data.

We were also interested in the behaviour and condition of pedestrians, comparing mainly the positive and negative manifestations related to age and gender. One of the most important factors is also the intoxication of pedestrians by alcohol or drugs (e.g., Fontaine \& Gourlet, 1997; Lee \& Abdel-Aty, 2005).

Contemporary research has also shown an interesting relationship between visibility and the number of accidents. Although it is not a specific accident type, approximately two thirds of pedestrian fatalities occur at night or under low-light conditions. The statistics from 2010 shows, that in the EU countries, 51\% of the pedestrian fatalities occurred at night. The rate for the Czech Republic was 47\% (Pace et al., 2012).

The next most frequently observed factor which we are interested in is the location of pedestrian collisions, especially in urban areas (Beck et al., 2007), and a certain location where collisions take place. Most of the accidents occurred in urban areas (Gitelman et al., 2012).

The main aim is to identify the characteristics of pedestrians and the environment and how they are associated with pedestrian accidents and its consequences. The information on the collision occurrence may help formulate and implement appropriate prevention measures.

\section{DATA AND METHODOLOGY}

The data analysed in this study were obtained from police crash reports in the Czech Republic. The analysis focuses on the period between 2008 and 2012. The crash record database used for the purpose of this paper contains records of all pedestrian collisions during the period for all the observed factors.

A frequency analysis focused on factors related to pedestrians themselves (pedestrian factors), i.e. age, gender, severity of injury, behaviour in accident situation, alcohol-impaired walking, pedestrian condition, and also roadway/environmental factors, i.e. type of collision participants, location, weather conditions, visibility or field of view (sight lines). Although in most studies these factors are usually evaluated independently, we tried to find conjunctions to provide a broad picture of concurrent characteristics of pedestrian accidents. For this purpose programmes for statistical analyses SPSS were used to run these operations (crosstabs). 
During the analysed period, there was the total of $\mathrm{N}=19454$ reported accidents involving pedestrians. The basic analysis was based on variables such as age, gender and severity of injury.

The age groups were divided into three categories - children (below 15 years of age), adults (15 - 64 years of age) and the elderly (seniors) (65 years and more). This categorization well reflects the results of the current research, where children and elderly pedestrians are supposed to be the most frequent and vulnerable participants of road accidents.

The classification of injuries comes out of the legislation of the Czech Republic and offers the following description: (1) death, (2) severe injury, (3) minor injury and (0) no injury. Minor injury is every injury which is not considered as severe injury and the treatment (including sick leave) is not required for this category (Decree of Ministry of Transport CR, 32/2001 Coll., S. 2, (d)). This classification is, in fact, based on the International Classification of injury (according to injury severity score/ISS): (a) lethal: the injuries are non-survivable even with immediate medical attention. Severe crushing injury and decapitation are examples. AIS: at least one level 6, non-survivable injury - ISS $=76$. (b) severe: the injuries pose a serious threat to the individuals' life and require immediate medical attention. AIS: at least one level 5, severe injury, or at least three regions with level 3 or 4 injuries - ISS 25 - 75. (c) moderate: injuries are survivable, but may require medical attention. Without medical attention the injuries could become severe. AIS: no more than two injuries with severity above 3 or 4 - ISS $=13$ - 24 (International Classification of Diseases-10 ${ }^{\text {th }}$ Edition).

Factors analysed in this paper involve: pedestrian factors: (a) age (b) gender (c) severity of injury (d) pedestrian condition (good condition, inattention, alcohol/drugs abuse, physical disabilities, suicidal behaviour, and other unspecified conditions), (e) pedestrian behaviour (in categories good/adequate and incorrect). roadway/environmental factors: (a) weather conditions (normal, foggy, light rainy, rainy, snowy, icy, windy, others), (b) visibility (day, twilight, or night with/without city lights), (c) field of view (good or poor caused by the construction or the surroundings, vegetation in the line of sight, etc.), (d) accident location (urban/rural areas) and more detailed description of the accident location and circumstances (at pedestrian crossing, at signalized or non-signalized pedestrian crossing, etc.).

\section{RESULT}

The study was performed on the whole sample of pedestrian collisions ( $\mathrm{n}=19454)$ recorded by police over the last five years (2008-2012) in the Czech Republic. The quantitative and percentage distribution is illustrated in the following charts.

\subsection{Age and gender}

The data describing the distribution of participants by gender were compared to the data from the National population census (2011) - it shows that there are approximately $48.9 \%$ of males and $51.1 \%$ of females in the whole population of the Czech Republic. The number of male pedestrian accident participants is $51.4 \%$ and $48.6 \%$ females in our sample, so there is no substantial difference.

Differences in total rate of designated age groups are more interesting. In the population of the Czech Republic there are $14.5 \%$ of population in the age group $0-14$ years, in comparison to $21 \%$ of pedestrians involved in accidents, $69.5 \%$ in the age group $15-64$ years (59\% pedestrians involved in accidents) and the group over the 64 years of age includes $16 \%$ of the population (20\% of pedestrians involved in accidents). Based on this data and in compliance with the foreign research mentioned above, children and the elderly seem to more often become the casualties of traffic accidents involving pedestrians. 
The distribution of pedestrian casualties according to age and gender is shown in Figure 1. As we can see, men seem to be involved in pedestrian accidents more often (except the category over 65 years of age), but the difference is not substantial.

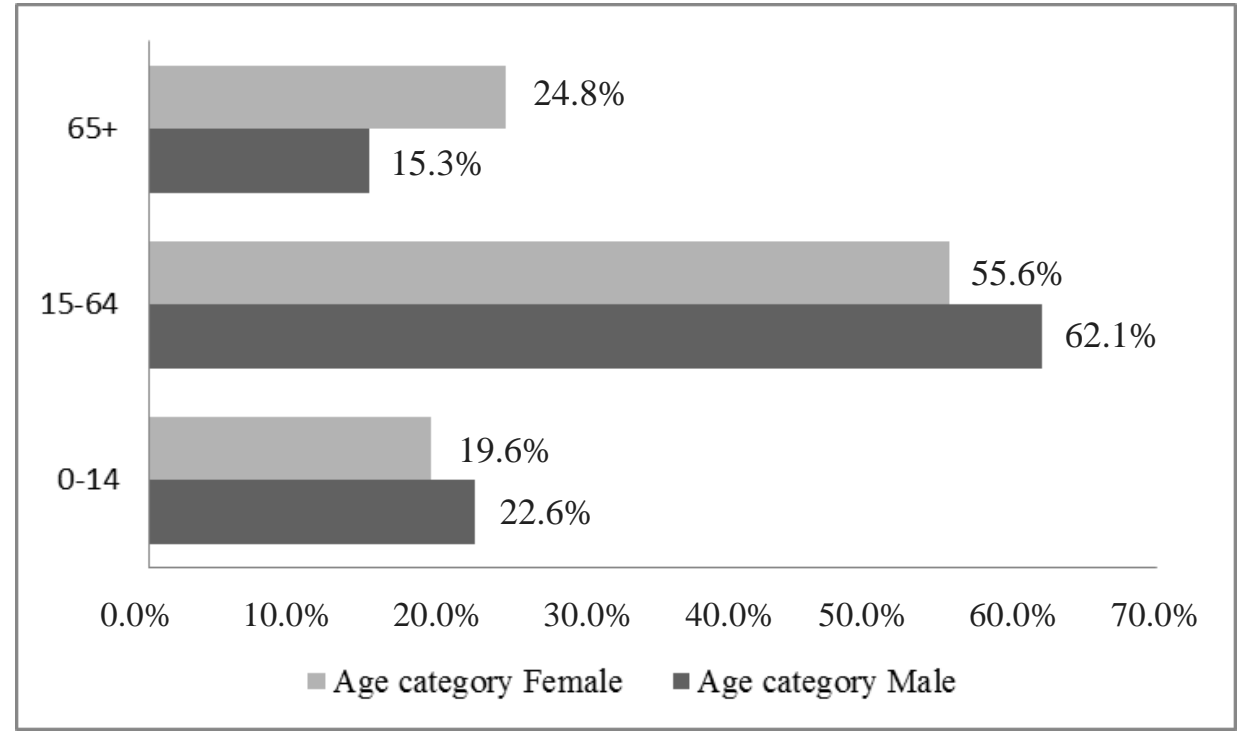

Figure 1: Distribution of the involved in accidents - age and gender.

\subsection{Severity of injury}

In terms of injury severity in our sample, the greatest amount of injured persons suffered from minor injuries (71.4\%), followed by severe injuries (17.8\%) and then death $(4.5 \%)$. $6.4 \%$ pedestrians had no injuries at all.

When taking into account the age groups (see Figure 2), the elderly suffered from fatal and severe injuries more frequently (7.0\% fatal and $25.5 \%$ severe injuries in this age group). On the contrary, within the age group $0-14$, the fatal consequences were present only in $0.7 \%$ cases, but on the other hand, minor injuries occurred within this age group very often (more than 80\%). In terms of injury severity, significant differences between men and women were not detected.

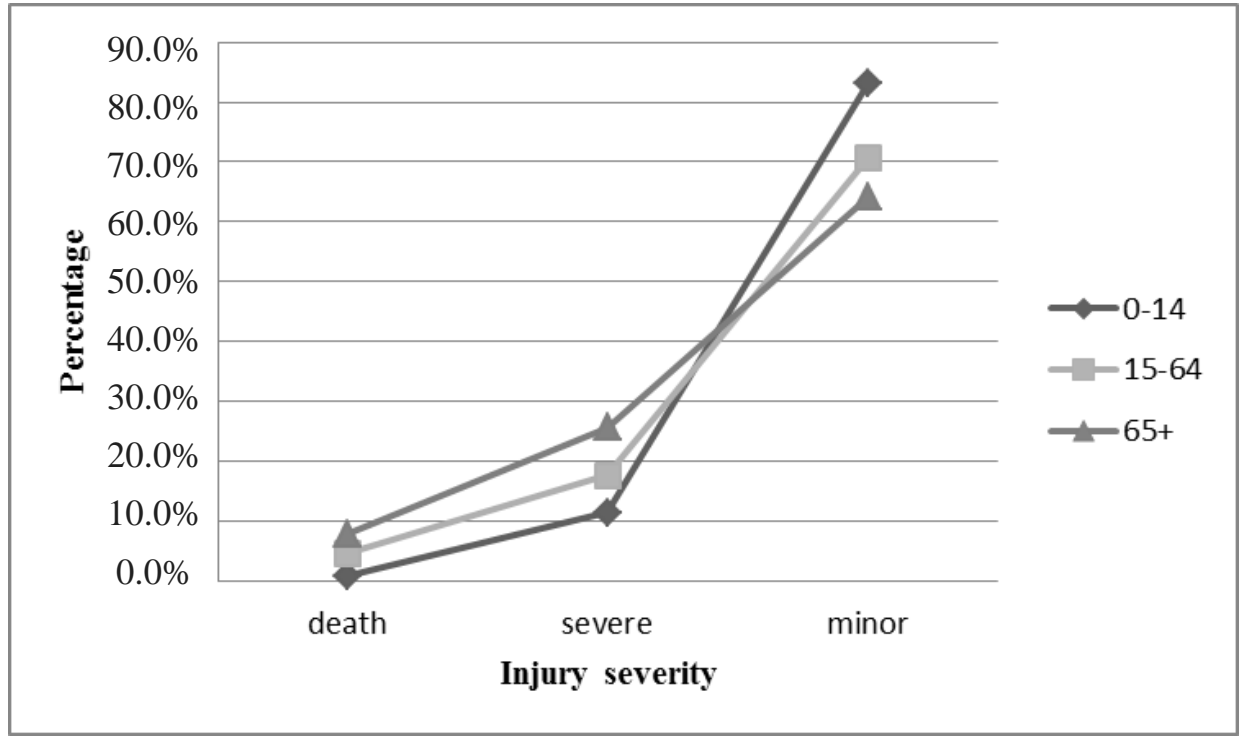

Figure 2: Age and injury severity. 


\subsection{Pedestrian condition}

It is not always possible to obtain exact data about pedestrian condition before the collision itself. Even police records usually do not contain detailed information, because it is usually very difficult to find out during the investigation what exactly was the pedestrian doing and what was running through his/her mind. For that reason, in quite a large amount of data (over 15\%) pedestrian condition is marked as unknown. Unfortunately, other categories are more or less vaguely defined - over $60 \%$ pedestrians were in a good condition, almost $12 \%$ pedestrians were marked as inattentive. The definition of inattention in police terminology means other activities but paying attention to other road users (which includes eating, drinking, smoking, using mobile phone, talking to another person while crossing the street, etc.). There were almost $30 \%$ of the accidents involving children (0-14 years of age) in this category (inattention). Alcohol-impaired walking was detected in $11 \%$ of all pedestrian collisions, especially in the population of the middle-aged men (24.5\%) and elderly men (nearly $10 \%)$. There were gender differences, too, e.g. female pedestrians were in a good condition in $71 \%$ of all cases, in comparison with male pedestrians (50\%). In other categories the numbers were unsubstantial. See Figure 3 for more.

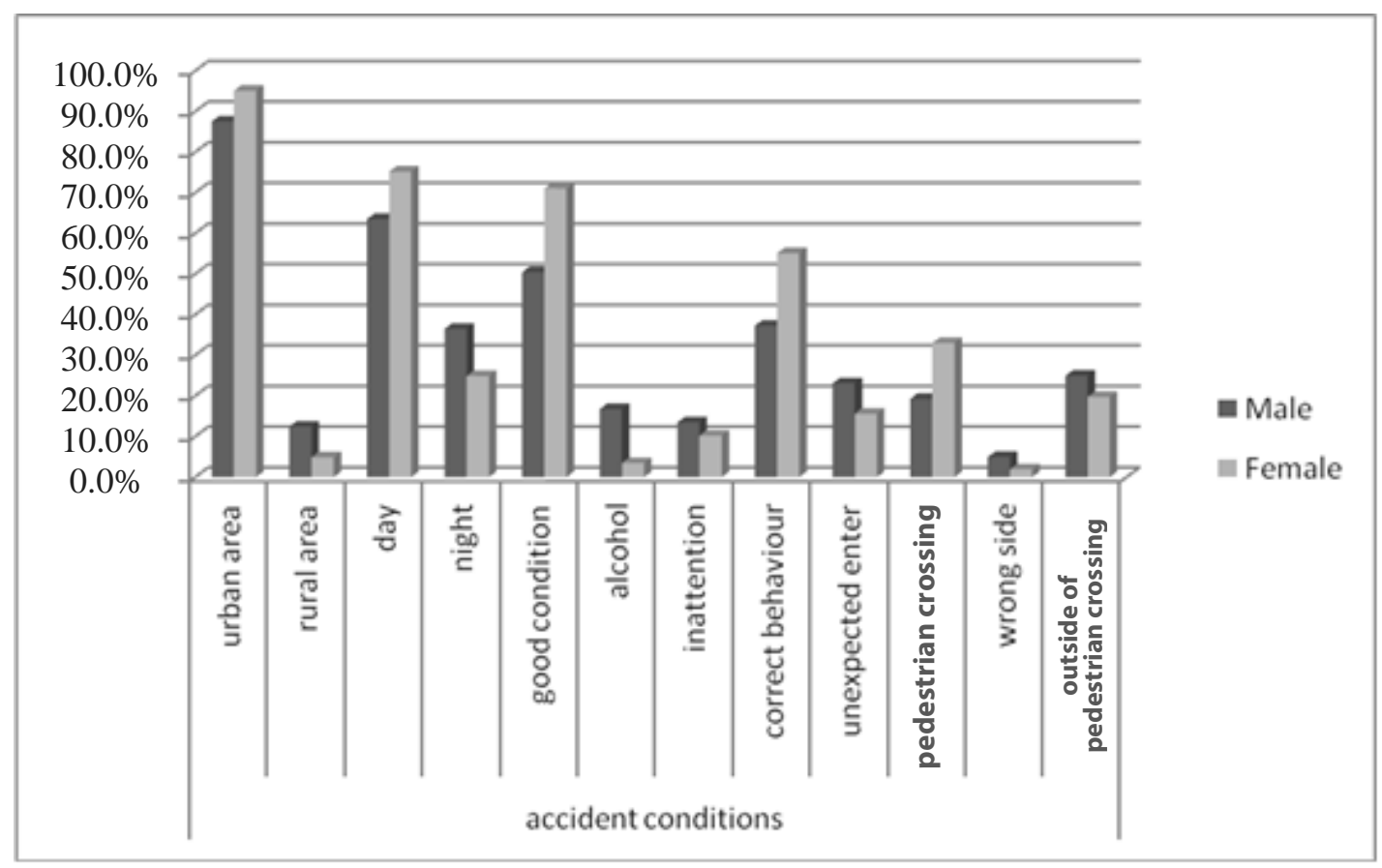

Figure 3: Gender and pedestrian condition/behaviour/accident location/visibility.

\subsection{Pedestrian behaviour}

Pedestrian behaviour was evaluated as correct in $45.5 \%$ cases. Just in the category pedestrian condition (see above), the pedestrian behaviour was marked as unknown in the substantial amount of the data (19\%). When looking at other categories, in $17 \%$ of all collisions, pedestrians unexpectedly stepped onto the road. The results worthy to notice occurred within the age group 0-14 (see Figure 4), where the correct behaviour was the lowest one of all age groups and concurrently the highest in all other monitored categories (i.e. unexpected enter from pavement in 37\%, crossing between parked vehicles in 10\% and crossing off a pedestrian crossing in 28\%). The difference between male and female pedestrian behaviour is shown in Figure 3. The correct behaviour was detected in 55\% of female and $36 \%$ of male pedestrians. That could indicate that women comply with traffic rules more responsibly than men. 


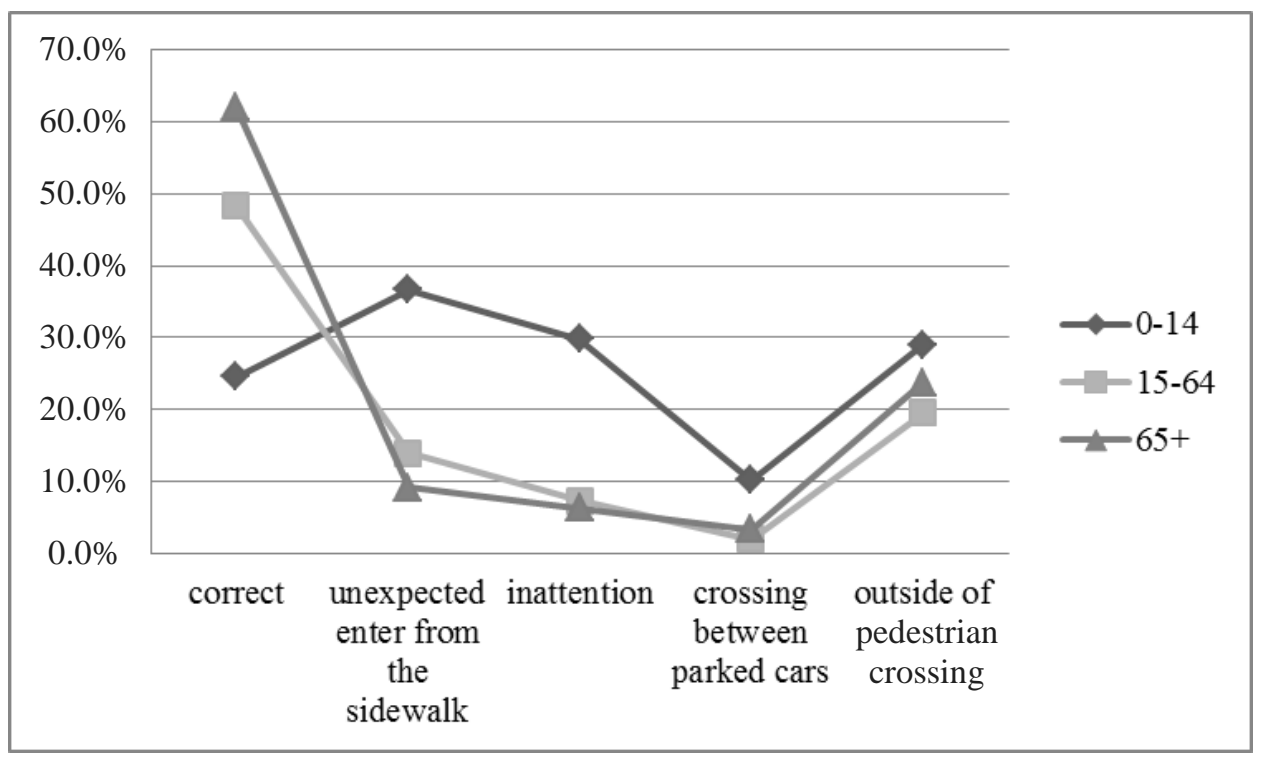

Figure 4: Age and pedestrian behaviour.

\subsection{Roadway/environmental factors}

Within the categories included in this observed factor (i.e. weather, visibility, field of view and accident location), there were no significant impacts to be found in relation with age, gender or severity of injury. More detailed analysis of the accident location shows that the greatest number of pedestrian accidents occur in urban areas $(91.2 \%$ in comparison to $8.8 \%$ in the rural areas), no substantial differences were found in relation to a certain accident location (at/off pedestrian crossing etc.) and age, gender and severity of injury in our sample.

\section{CONCLUSIONS AND RECOMMENDATIONS}

The study analysed the police crash records of pedestrian collisions in the period between 2008 and 2012 in the Czech Republic. The aim of this study was to identify and refer to critical points of this type of accidents. Our research results are quite consistent with earlier foreign research.

First, children and elderly are the most vulnerable road users, which is confirmed by our study.

Both these groups become casualties of pedestrian collisions more often than the rest of the population and, therefore, safety measures should be put in place to better support their protection. Other factors related to pedestrian accidents, such as pedestrian condition and behaviour, field of view, occurred in relationship with some of age and gender groups.

Whereas the elderly suffer from fatal and severe injuries most of all participants, the greatest amount of their collisions occur when crossing the road at pedestrian crossings and their behaviour is evaluated as good as well as their condition. So they seem to be less willing to break the traffic rules and tend to behave more responsibly. The reason for the higher frequency and also fatality of their collisions can be added to their higher physical fragility and also motoric and perception limitations and disabilities related to age. Being aware of their decreased locomotive ability, elderly pedestrians integrate their longer crossing time into the decision-making process (Lobjois \& Cavallo, 2007). 
In comparison to the other age groups, children suffer from minor injuries most often, their fatality is one of the lowest, and collisions occur most frequently when crossing between parked vehicles (including bus stops) and on unprotected road sections without pedestrian crossing. Their behaviour is described as incorrect the most frequently of all age groups, whereas boys are prone to risky behaviour more often than girls while crossing the road. Almost one third of all accidents including a child participant were ascribed to child's inattention.

When trying to find the explanation for the differences in pedestrian behaviour related to age, several studies including this paper were searching for an answer. The possible explanation, for example, is that middle-aged pedestrians have usually good perceptive skills and a high walking speed, but they are more conservative than their younger counterparts, thus likely to improve their safety (Zhuang \& Wu, 2011). On the other hand, young people have more positive attitude and intention towards committing violations than adults and report more errors and lapses than adults (Díaz, 2002).

Within the whole sample of pedestrian collisions, the study identified specific gender differences. First, males seem to participate in pedestrian accidents more often than females and also alcohol-impaired walking was detected more frequently, especially in the population of middle-aged and elderly males. Women also seem to comply with traffic rules more responsibly than men, their behaviour is evaluated as correct and their condition as good more frequently than men's. According to Díaz (2002), higher probability of becoming the male casualty of pedestrian collision can be explained by the fact that males violate traffic rules more frequently.

The weather and the accident location have not been proved as a relevant factor influencing pedestrian accidents. However, weather as a potential influencing factor should not be rejected as it needs to be assessed along with other relevant factors, not independently (Edwards, 1996).

In this study, current findings in selected aspects concerning to pedestrian accidents in the Czech Republic were confirmed.

\section{ACKNOWLEDGEMENT:}

The article was produced thanks to the institutional support for the long-term conceptual research institute development.

\section{REFERENCES}

Al-Shammari, N., Bendak, S., Al-Gadhi, S., 2009. In-Depth Analysis of Pedestrian Crashes in Riyadh. Traffic Injury Prevention, 10 (6), pp. 552-559.

Beck, L. F., Paulozzi, L. J., Davidson, S. C., 2007. Special Report from the CDC - Pedestrian fatalities, Atlanta Metropolitan Statistical Area and United States, 2000-2004. Journal of Safety Research, 38 (6), pp. 613-616.

Criminal Code of Czech Republic. Law 140/1961, § 89, b 7.

Díaz, E. M., 2002. Theory of planned behavior and pedestrians' intentions to violate traffic regulations. Transportation Research Part F: Traffic Psychology and Behaviour, 5 (3), pp. 169-175.

Edwards, J. B., 1996. Weather-related road accidents in England and Wales: a spatial analysis. Journal of Transport Geografy, 4 (3), pp. 201-212. 
Fontaine, H., Gourlet, Y., 1997. Fatal pedestrian accidents in France: a typological analysis. Accident Analysis and Prevention, 29 (3), pp. 303-312.

Gitelman, V., Balasha, D., Carmel, R., Hendel, L., Pesahov, F., 2012. Characterization of pedestrian accidents and an examination of infrastructure measures to improve pedestrian safety in Israel. Accident Analysis and Prevention, 44 (1), pp. 63-73.

Holland, C., Hill, R., 2007. The effect of age, gender and driver status on pedestrians' intentions to cross the road in risky situations. Accident Analysis and Prevention, 39 (2), pp. 224-237.

International Classification of Diseases $-10^{\text {th }}$ Edition.

Lee, Ch., Abdel-Aty, M., 2005. Comprehensive analysis of vehicle-pedestrian crashes at intersections in Florida. Accident Analysis and Prevention, 37 (4), pp. 775-786.

Lobjois, R., Cavallo, V., 2007. Age-related differences in street-crossing decisions: The effects of vehicle speed and time constraints on gap selection in an estimation task. Accident Analysis and Prevention, 39, pp. 934-943.

Pace, J. F. et al., 2012. Basic Fact Sheet "Pedestrians", Deliverable D3.9 of the EC FP7 project DaCoTA.

PACTS, 2013. Stepping out: pedestrian casualties: an analysis of the people and the circumstances. Parliamentary Advisory Council for Transport Safety (PACTS). 29 p.

Prato, C. G., Gitelman, V., Bekhor, S., 2012. Mapping patterns of pedestrian fatal accidents in Israel. Accident Analysis and Prevention, 44 (1), pp. 56-62.

The Order of Ministry of Transport CR, 32/2001 Sb., § 2, d.

The Police Statistic of Czech Republic, Annual 2012.

WHO, 2013. Pedestrian safety: a road safety manual for decision-makers and practitioners. World Health Organization. 114 p.

Yannis, G., Papadimitriou, E., Evgenikos, P., 2011. About Pedestrian Safety in Europe. Advances in Transportation Studies, 24, pp. 5-14.

Zegeer, Ch.V., Bushell, M., 2012. Pedestrian crash trends and potential countermeasures from around the world. Accident Analysis and Prevention, 44 (1), pp. 3-11.

Zhuang, X., Wu, Ch., 2011. Pedestrians' crossing behaviors and safety at unmarked roadway in China. Accident Analysis and Prevention, 43 (6), pp. 1927-1936. 